\title{
Identification of Quantitative Trait Loci Controlling Symptom Development During Viral Infection in Arabidopsis thaliana
}

\author{
Ophélie Sicard, ${ }^{1}$ Olivier Loudet, ${ }^{2}$ Joost J. B. Keurentjes, ${ }^{3}$ Thierry Candresse, ${ }^{1}$ Olivier Le Gall, ${ }^{1}$ \\ Frédéric Revers, ${ }^{1}$ and Véronique Decroocq ${ }^{1}$ \\ ${ }^{1}$ UMR Génomique, Diversité et Pouvoir Pathogène, Institut National de la Recherche Agronomique (INRA)-Université \\ Bordeaux 2, IBVM, , BP81, 33883 Villenave d'Ornon, France; '2Station de Génétique et d'Amélioration des Plantes, INRA, \\ route de Saint Cyr, 78026 Versailles cedex; France ${ }^{3}$ Laboratory of Plant Physiology and Genetics, Wageningen University, \\ NL-6703 BD, Wageningen, The Netherlands
}

Submitted 24 July 2007. Accepted 12 October 2007.

In compatible interactions between plants and viruses that result in systemic infection, symptom development is a major phenotypic trait. However, host determinants governing this trait are mostly unknown, and the mechanisms underlying it are still poorly understood. In a previous study on the Arabidopsis thaliana-Plum pox virus (PPV) pathosystem, we showed a large degree of variation in symptom development among susceptible accessions. In particular, Cvi-1 (Cape Verde islands) accumulates viral particules but remains symptomless, Col-0 (Columbia) sometimes shows weak symptoms compared with Ler (Landsberg erecta), which always shows severe symptoms. Genetic analyses of Col $\times$ Ler and Cvi $\times$ Ler F2 and recombinant inbred line (RIL) populations suggested that symptom development as well as viral accumulation traits are polygenic and quantitative. Three of the symptom quantitative trait loci (QTL) identified could be confirmed in near-isogenic lines, including PSII (PPV symptom induction 1), which was identified on the distal part of chromosome 1 in both RIL populations. With respect to viral accumulation, several factors have been detected and, interestingly, in the Col $\times$ Ler population, two out of three viral accumulation QTL colocalized with loci controlling symptom development, although correlation analysis showed weak linearity between symptom severity and virus accumulation. In addition, in the Cvi $\times$ Ler RIL population, a digenic recessive determinant controlling PPV infection was identified.

Two basic types of interaction exist between a virus and its host plant: i) a compatible interaction associated with plant invasion and viral multiplication, followed in most cases by the induction of symptoms, and ii) an incompatible interaction with no or only limited viral multiplication or movement in the host plant. Plant-virus interactions are relatively well documented, but efforts have so far focused on resistance (or resistancebreaking) mechanisms and on the identification of their genetic determinants. Conversely, the one or more mechanisms controlling symptom development in plants are still largely unknown,

Corresponding author: V. Decroocq; E-mail: decroocq@bordeaux.inra.fr; Fax: +0033-557122384.

* The $\boldsymbol{e}$-Xtra logo stands for "electronic extra" and indicates supplemental material is published online. Three additional figures are available online. so that only a very patchy picture of the interaction between a plant and a virus can be presented.

Genetic factors controlling symptoms in compatible plantvirus interactions have been studied both on the host and on the pathogen side. In the host, symptom development has been reported to be controlled by either monogenic or polygenic genetic determinants, dominant or recessive. For instance, the symptom determinants in the pathosystems of $A$. thaliana and Tobacco ringspot nepovirus, Spring beauty latent bromovirus, or Turnip mosaic virus (TuMV) were shown to be monogenic and semidominant traits (Lee et al., 1996; Fujisaki et al. 2004; Kaneko et al. 2004). Similar monogenic controls, however recessive, were reported in the case of the symptom development during infection of A. thaliana by Beet curly top virus and Tobacco mosaic virus (Dardick et al. 2000; Park et al. 2002). On Nicotiana tabacum infected with Cucumber mosaic virus (CMV), the genetic determinism of symptoms was shown to be digenic and recessive (Takahashi and Ehara 1993). Finally, even more complex mechanisms controlling symptom development have been observed, with multiple contributing genes. Such polygenic control is the case with the A. thaliana-Cauliflower mosaic virus pathosystem, for which three distinct quantitative trait loci (QTL) have been identified (Callaway et al. 2000). Similarly, quantitative symptom determinants have also been identified in several other pathosystems such as pepper-CMV (Ben Chaim et al. 2001), tomato-Tomato yellow leaf curl virus (Zamir et al. 1994), and Prunus davidiana-Plum pox virus (PPV) (Decroocq et al. 2005). However, none of these genes have been cloned yet.

The viral determinants involved in symptom development or controlling symptom severity have frequently been observed to be inhibitors of the plant RNA silencing antiviral defense system (Dunoyer and Voinnet 2005; Vance and Vaucheret 2001; Voinnet 2001). Such is, for example, the case for P1/HC-Pro, a silencing suppressor of the potyvirus TuMV, which appears to exert its effect through interference with the miRNA-controlled developmental pathway, thus inducing developmental abnormalities and symptom development in the host plant (Dunoyer et al. 2004; Kasschau et al. 2003).

As detailed above, the model plant $A$. thaliana has often been used for the identification of plant genetic factors involved in plant-pathogen interactions. Recently, we have shown that PPV, the causal agent of sharka disease in stone fruit trees, is able to infect $A$. thaliana (Decroocq et al. 2006). A wide range of interaction outcomes were observed, depending on the host genotype 
or on the particular PPV isolate being used: resistance at the inoculation point (with no local virus multiplication detectable), resistance to systemic viral invasion (with viral accumulation in the inoculated leaves but not distantly from the inoculation point), and full systemic susceptibility. Depending on the particular combination between a susceptible Arabidopsis accession and the viral isolate, a broad range of reactions were observed, from the absence of symptoms to severe symptom development. Initial efforts toward the identification of one or more genetic factors underlying this variability in symptom development indicated that, when confronted by the PPV-R (Rankovic) isolate, the difference in response between the Col-0 (Columbia) accession (mild symptoms) and the Ler (Landsberg erecta) accession (severe symptoms) is controlled by at least two loci and segregates as a quantitative trait (Decroocq et al. 2006).

The focus of the present study was the further characterization of plant determinants controlling symptom development as well as PPV accumulation in A. thaliana. For that, a quantitative genetic analysis was performed within the $\mathrm{Col} \times \mathrm{Ler}$ F2 and recombinant inbred line (RIL) populations. A similar approach was followed using populations derived from the Ler parent and Cvi-1 (Cape Verde islands), an accession that shows only extremely limited symptom development. The use of near-isogenic lines (NILs) allowed the confirmation of QTL identified during the quantitative analysis. Finally, a digenic and recessive resistance has been identified following the analysis of the Cvi $\times$ Ler population, in which each parent contributes one resistance genetic determinant.

\section{RESULTS}

\section{Symptom occurrence}

in Arabidopsis thaliana accessions infected with PPV-R.

To identify the genetic determinants controlling symptom development and viral accumulation during PPV infection in A. thaliana, a detailed phenotypic characterization of the most promising Arabidopsis accession and viral isolate combinations identified by Decroocq and associates (2006) was performed. Two parameters were scored, i.e., the virus accumulation in infected plants and symptom development. Approximately 2 weeks after inoculation up to 21 days postinoculation (dpi), each inoculated plant was rated visually for symptoms (SYMP). A score was given to the obtained phenotype following a notation scale between 0 (no symptom but viral accumulation) and 4 (Fig. 1). At three weeks postinoculation, virus accumulation (ACC) was determined by optical density and a
1:100 dilution was performed in order to have a better value distribution. The PPV-R isolate was selected because it results in a wide range of infection phenotypes in different accessions and because an infectious cDNA clone of this isolate is available, thus eliminating potential problems associated with genetic drift of the isolate between experiments.

Upon inoculation with PPV-R, the three A. thaliana accessions Ler, Col-0, and Cvi-1 differed substantially in symptom development and virus accumulation. The Ler accession showed the most consistent response. All of the 99 inoculated plants developed severe symptoms $(\mathrm{SYMP}=3.0 \pm 0)$ and heavily accumulated the virus in noninoculated inflorescence tissues (ACC $=40.7 \pm 8.6 \%$ of the positive Nicotiana benthamiana control). Plants of the Col-0 accession showed a more variable phenotype but, overall, expressed much milder symptoms than Ler $(\mathrm{SYMP}=1.29 \pm 1.25)$. Irrespective of the presence of symptoms, all inoculated Col plants accumulated PPV-R but with substantial variation in the accumulation level (ACC of $40.0 \pm 35 \%$ of positive control). There was only a weak correlation between symptom severity and viral accumulation $\left(R^{2}\right.$ of 0.42). The Cvi accession showed an extremely variable response. Of 96 inoculated Cvi plants, 69 did not accumulate the virus in the noninoculated tissues and, therefore, were considered to be resistant. In all other experiments performed, a similar fraction of the inoculated Cvi plants were found to fail to accumulate the virus (discussed below). This observation was in contrast with our previous data (Decroocq et al. 2006), in which we reported Cvi to be a susceptible, symptomless host of PPV-R. This discrepancy may be explained by the fact that the noninfected plants observed by Decroocq and associates (2006) were considered as inoculation escapes and were therefore discarded from further analyses.

Of the remaining $27 \mathrm{Cvi}$ plants in which viral accumulation was detected, only four developed weak symptoms (SYMP = $1.6 \pm 0.75)$, all other plants remained symptomless. In all experiments performed in this study, these four Cvi plants were the only ones that developed symptoms upon PPV-R inoculation. The 27 PPV-R-accumulating plants had a mean ACC value of $42.4 \pm 44.6 \%$ of the positive control and could be separated in two groups: 12 plants accumulating the virus at high levels $(\mathrm{ACC}=86.0 \pm 30 \%$ of control $)$ and 15 accumulating the virus at a low levels $(\mathrm{ACC}=7.5 \pm 3 \%)$. The four plants that showed symptoms belong to the high-accumulating group.

It should be noted that the SYMP scores are relatively stable from one experiment to another but that the ACC values can vary substantially. This variation may be a consequence of
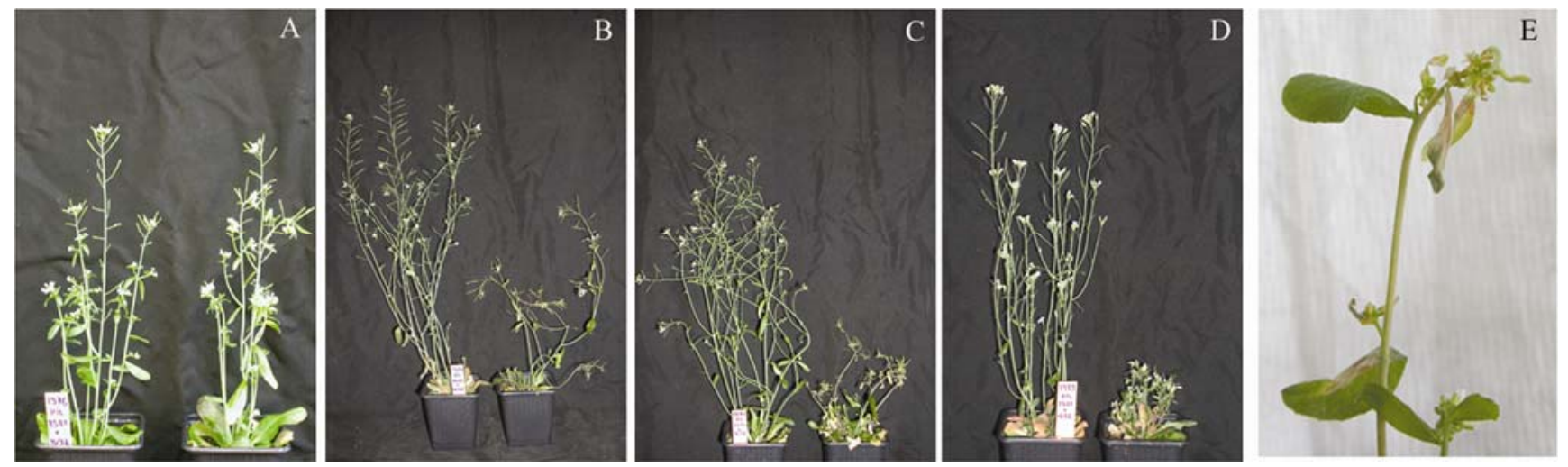

Fig. 1. Various degrees of symptom severity in the Columbia $\times$ Landsberg erecta recombinant inbred line population. Similar phenotypes were observed in the Cape Verde islands $\times$ Ler population. Healthy plants are on the left of each picture and inoculated plants, on the right. A, Plants exhibiting symptoms scoring 1 , one floral hamp with top incurvation, $\mathbf{B}$, plants showing symptoms scoring 2, a majority of the floral hamps are curved but no or very low reduction size, $\mathbf{C}$, plants showings a phenotype scored 3 with all hamps presenting incurvation and a strong reduction of their size, and $\mathbf{D}$, plant corresponding to a symptom score of 4, with heavily reduced hamp development and stunted hamp. E, Details of a symptomatic floral hamp. 
changes in environmental conditions, which may influence the virus accumulation in the Arabidopsis host plants or even in the $N$. benthamiana positive control. However, when evaluated simultaneously, the difference in response of the various accessions was always consistent and reproducible in various experiments. PPV-R accumulation was always found to be higher in Ler than in Col (discussed below), while comparable average accumulation levels were reached in Ler and in Cvi (though with a larger standard deviation for Cvi). However, the highaccumulating subpopulation of Cvi clearly reached higher viral titers than any other evaluated plant, despite the fact that these plants displayed no or only mild symptoms.

\section{Phenotypic variation in $\mathrm{F} 2$ populations.}

In order to determine the genetic basis controlling the SYMP and ACC traits, F2 populations derived from the $\mathrm{Col} \times \mathrm{Ler}$ and Ler $\times$ Cvi crosses were inoculated with PPV-R, and SYMP and ACC were scored on individual F2 plants. Both traits showed a normal distribution (Supplemental Fig. S1) in the F2 $\mathrm{Col} \times$ Ler population. In the Cvi $\times$ Ler F2 population, ACC values were also normally distributed, but this was not the case for the SYMP trait. In addition, no virus could be detected in the inflorescence tissues of 12 of the 110 inoculated $\mathrm{F} 2$ plants. Given the $100 \%$ infection rate observed in the plants of the Col $\times$ Ler population and the resistance phenotype observed in part of the Cvi PPV-R-inoculated plants, these 12 plants are unlikely to be inoculation escapes but should rather be considered as resistant to PPV-R. The analysis of this resistance is described below.

For both traits, transgressive segregation was observed in all populations. In particular, a substantial number of plants (between one-quarter and one-third) of the F2 populations developed severe symptoms (Fig. 1, score 4 on the symptom scale), whereas such symptom intensity was never observed in any of the parents. These data suggest that SYMP and ACC are quantitative traits controlled by multiple genes.

\section{Correlation, genetic effect, and heritability.}

RIL populations derived from the Col $\times$ Ler (Lister and Dean 1993) and Ler $\times$ Cvi (Alonso-Blanco et al. 1998b) crosses were inoculated with PPV-R, and SYMP and ACC were scored. For each RIL, the values obtained were averaged over five $(101 \mathrm{Col} \times$ Ler RILs) and four $(156$ Cvi $\times$ Ler RILs) individual plants, respectively. For the $\mathrm{Col} \times \mathrm{Ler}$ population, $100 \%$ of the inoculated RILs showed viral accumulation. For the Cvi $\times$ Ler population, 24 of the 156 RILs did not show detectable virus accumulation and were classified as resistant to PPV-R infection. Resistance is defined in this study by the absence of the virus in noninoculated leaves and, therefore, the absence of symptoms. These RILs were used for the analysis of the resistance (discussed below) but were excluded from further analysis of the SYMP and ACC traits, since the effect of the virus on its host was biased. The correlation between SYMP and ACC scores was evaluated for each RIL population. In both crosses, the symptom severity showed only weak correlation with viral accumulation (Supplemental Fig. S2). Coefficients calculated with the statistical software $\mathrm{R}$ showed average $\left(0.48, P=9.71 \times 10^{-7}\right)$ to weak $\left(0.23, P=9.2 \times 10^{-3}\right)$ correlation in the $\mathrm{Col} \times$ Ler and Ler $\times$ Cvi populations, respectively. For example, in both populations, RILs could be identified that have severe symptoms (SYMP score $=3$ ) with ACC scores of between 25 and $90 \%$ of the positive control. Conversely, other RILs presented weak or no symptoms but a high virus titer.

Fischer's F-values and heritabilities, obtained from the analysis of variance (ANOVA) on RIL population data, are presented in Table 1 . The genetic effect is highly significant for both traits $(P(f)<0.0001)$ in the two populations. Heritabilities of 43 and $54 \%$ were obtained in the Col $\times$ Ler population for the ACC and SYMP traits, respectively, indicating that approximately half of the variation observed in the phenotype can be explained by genotypic differences. For the Ler $\times$ Cvi cross, heritabilities were even higher for both traits $(75$ and $65 \%$ for ACC and SYMP, respectively).

\section{QTL analysis.}

The QTL analyses were performed on the Col $\times$ Ler and Cvi $\times$ Ler RIL populations and using the quantitative values for the SYMP and ACC traits described above as phenotypic data.

Concerning the $\mathrm{Col} \times \mathrm{Ler}$ population, three QTL were detected for the ACC trait on chromosomes 1 (ACC1), 3 (ACC3), and 5 (ACC5), respectively (Table 2, Fig. 2). The Ler alleles of the $A C C 1$ and $A C C 3$ QTL increased ACC values (positive additive effect), while the Ler allele of the ACC5 QTL decreased ACC values (negative additive effect). The occurrence of multiple QTL with opposite additive effect segregating independently is expected to cause transgression above the parental Ler phenotypic value (Table 2). The percentage of phenotypic variance explained by each QTL ranged from 9 to $17 \%$. Two QTL were found to contribute to the SYMP trait explaining respectively 13 (PSI1.1, PPV symptom induction, located on chromosome 1) and $17 \%$ (PSI3, located on chromosome 3 ) of the phenotypic variance. A weak epistatic interaction, significant at $\alpha=0.05$, was detected between these two QTL. PSI1.1 maps on top of chromosome 1 , near the nga59 marker. This position is very close to that identified for the $A C C 1$ QTL (marker ve006), being only $12 \mathrm{cM}$ distant. The other SYMP QTL, PSI3, colocalizes with the ACC3 QTL on chromosome 3 , since it is linked to the same marker, $\mathrm{m} 424$.

In the Cvi $\times$ Ler population, only one QTL for the ACC trait could be identified, ACC4 on chromosome 4, with a negative additive effect (ACC values of Cvi are greater than those of Ler). For the SYMP trait, because of the nonnormal distribution of the data in the Cvi $\times$ Ler F2 and RIL populations, a Kruskal-Wallis rank-sum test was performed. Results identified three contributing loci on chromosomes 1 (PSI1.2), 2 (PSI2), and 5 (PSI5) (Table 2 and Fig. 2), with explained variances of 13.5, 5.6, and 9.2\%, respectively. PSII.2 and PSI2 had positive additive effect (SYMP values of Ler are greater than that of Cvi) while PSI5 had a negative additive effect (SYMP values

Table 1. Phenotypic variation for viral accumulation (ACC) and symptom development (SYMP) in recombinant inbred line (RIL) populations

\begin{tabular}{lcccccc}
\hline Trait & Columbia $(\mathbf{C o l})$ mean & Landsberg erecta $($ Ler) mean & RIL mean & RIL range (min to max) & Fischer's F $^{\text {a }}$ & Heritability (\%) \\
\hline ACC & $40.34( \pm 35.8)$ & $75.16( \pm 19.7)$ & 58.01 & 13.01 to 98.8 & $3.42^{* * *}$ & 43 \\
SYMP & $1.29( \pm 1.25)$ & $3.4( \pm 0.84)$ & 2.94 & 0.25 to 4 & $4.73^{* * *}$ & 54 \\
\hline Trait & Cape verde islands $($ Cvi) mean & Ler mean & RIL mean & RIL range (min to max) & Fischer's F & Heritability $(\%)$ \\
\hline ACC & $53.56( \pm 11.54)^{\mathrm{b}}$ & $37.73( \pm 6.9)$ & 34.31 & 14.37 to 86.52 & $9.48^{* * *}$ & 75 \\
SYMP & 0 & 3 & 1.11 & 0.25 to 4 & $6.24^{* * *}$ & 65 \\
\hline
\end{tabular}

$\mathrm{a} * * *=$ significant at the $0.01 \%$ level.

${ }^{\mathrm{b}}$ Mean calculated with infected plant (with virus accumulation). 
Table 2. Quantitative trait loci (QTL) controlling viral accumulation (ACC) and symptom development (SYMP) in the Columbia (Col) $\times$ Landsberg erecta $(\mathrm{Ler})$ and in the Cape verde islands $(\mathrm{Cvi}) \times$ Ler recombinant inbred line populations

\begin{tabular}{|c|c|c|c|c|c|c|c|}
\hline Trait & Chr $^{\mathbf{a}}$ & QTL name & Nearest marker & LOD score & Additive $^{b}$ & $\mathbf{P E V}^{\mathbf{c}}$ & \\
\hline \multicolumn{8}{|c|}{ QTL controlling symptom and viral accumulation in the $\mathrm{Col} \times \mathrm{L}$ er population after infection with the Plum pox virus Rankovic (PPV-R) isolate } \\
\hline \multirow[t]{3}{*}{ SYMP } & 1 & PSI1.1 & nga59 & 3.7797 & -0.3514 & 13.15 & \\
\hline & 3 & PSI3 & $\mathrm{m} 424$ & 4.4367 & -0.439 & 17.4 & \\
\hline & & & nga59 $\times$ m424 & $0.0339^{d}$ & 4.65 & & \\
\hline \multirow[t]{3}{*}{ ACC } & 1 & $\mathrm{ACC} 1$ & ve006 & 2.8513 & -5.7044 & 9.66 & \\
\hline & 3 & ACC 3 & $\mathrm{~m} 424$ & 4.2848 & -8.3012 & 15.07 & \\
\hline & 5 & ACC5 & nga139 & 3.287 & 7. 5449 & 16.59 & \\
\hline Trait & CHR & QTL name & Nearest marker & LOD score & Additive $^{b}$ & $\mathbf{P E V}^{\mathrm{c}}$ & \\
\hline \multicolumn{8}{|c|}{ QTL controlling viral accumulation in the Cvi $\times$ Ler population after PPV-R infection } \\
\hline ACC & 4 & ACC4 & EC.306L & 3.41 & -0.0583 & 11.84 & \\
\hline Trait & CHR & QTL name & Nearest marker & Mean rank Ler & Mean rank Cvi & $\mathbf{P E V}^{\mathbf{c}}$ & $\mathbf{K}^{\mathbf{e}}$ \\
\hline \multicolumn{8}{|c|}{ QTL controlling symptom development in the Cvi $\times$ Ler population after PPV-R infection } \\
\hline \multirow[t]{3}{*}{ SYMP } & 1 & PSI1.2 & PVV4 & 1.65495 & 0.773522 & 13.5 & $* * * * * *$ \\
\hline & 2 & PSI2 & FD.150C & 1.49876 & 0.894489 & 5.6 & $* * *$ \\
\hline & 5 & PSI5 & GH.117C & 0.876126 & 1.6423 & 9.2 & $* * * *$ \\
\hline
\end{tabular}

${ }^{\mathrm{a}} \mathrm{CHR}=$ chromosome; $\mathrm{LOD}=$ logarithm of the odds ratio.

${ }^{\mathrm{b}}$ In the $\mathrm{Col} \times$ Ler population, a negative value indicates the contribution of the Ler parent and in the Cvi $\times$ Ler population, this information is given by a positive value.

${ }^{\mathrm{c}}$ Percentage of variance (PEV) explained by the QTL.

${ }^{\mathrm{d}}$ Value given is the $P$ value instead of the LOD score, at the 5\% level.

${ }^{\text {e }} P$ value associated with the Kruskal-Wallis test: $* * *=0.01 * * * *=0.005$ and $* * * * * *=0.005$.

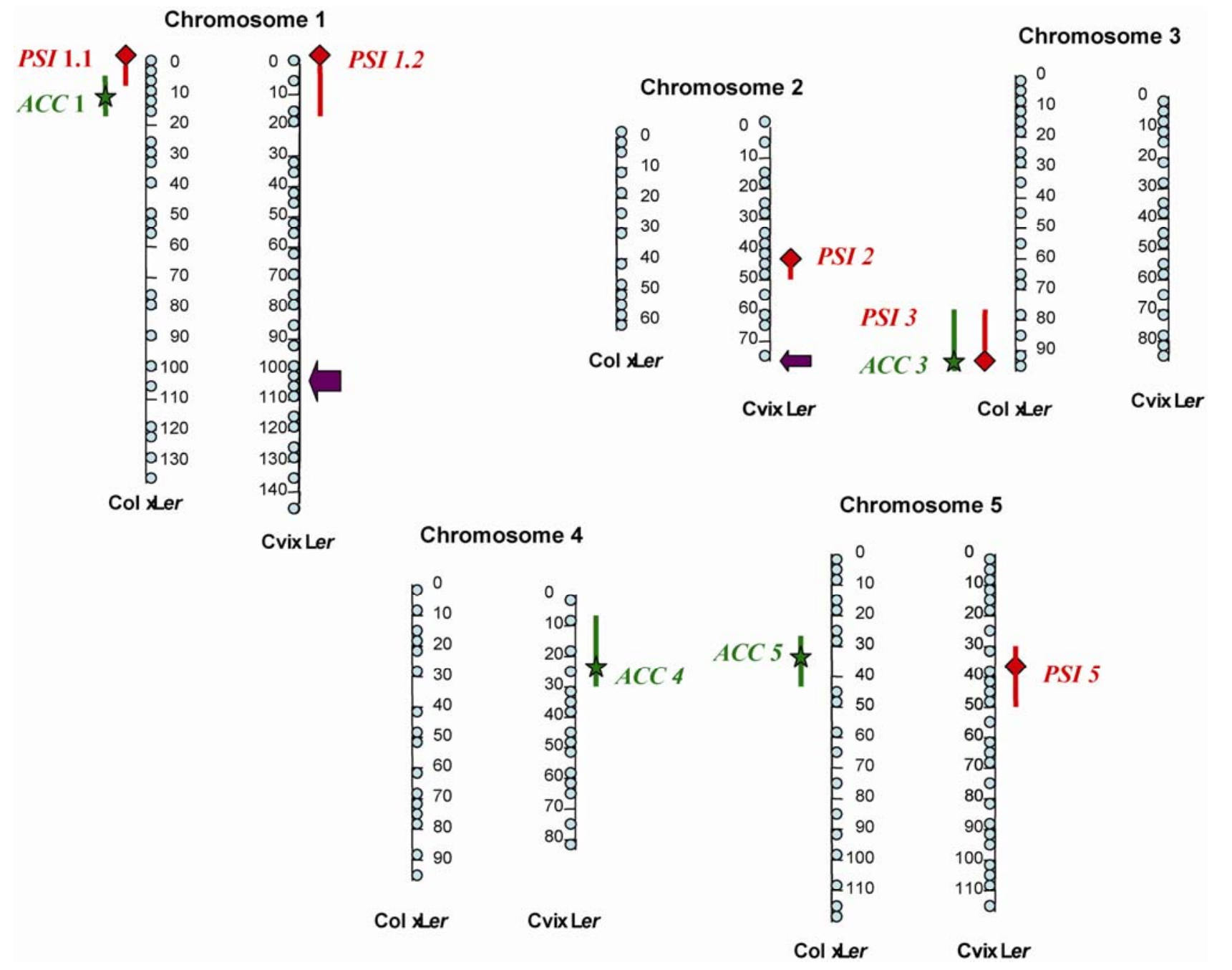

Fig. 2. Columbia $(\mathrm{Col}) \times$ Landsberg erecta (Ler) (left chromosome) and Cape Verde islands $(\mathrm{Cvi}) \times$ Ler (right chromosome) genetic maps showing quantitative trait loci (QTL) for symptom severity (red square) and virus accumulation (green star). Vertical lines associated with each QTL denote the 2-LOD (logarithm of the odds ratio) support interval. Approximate positions of resistance genes are indicated with a purple arrow. Units shown are in centimorgans, and markers used to construct the genetic maps are depicted as blue closed circles. 
of Cvi are greater than those of Ler). Interestingly, PSII.2, cosegregating with the PVV4 marker, colocalizes with the PSI1.1 QTL detected in the Col $\times$ Ler RIL population. However, despite colocalization and similarity in additive effect, we cannot rule out, at this stage, that PSII.1 and PSI1.2 are two distinct loci.

\section{QTL confirmation and fine mapping using NILs.}

To confirm and fine-map the PSII QTL located on top of chromosome 1, seven NILs (Keurentjes et al. 2007), each containing a different Cvi introgression in a Ler background, were used (Fig. 3). As PSII is genetically linked to the PVV4 marker, LCN1-2.3, LCN1-2.4, and LCN1-2.5 should not contain the Ler allele of PSI1, whereas LCN1-2.10, LCN1-2.11, LCN1-2.12, and LCN1-2.16 should. Indeed, after inoculation of this set of NILs by PPV-R, LCN1-2.3, LCN1-2.4, and LCN1-2.5 did not develop any symptoms, despite the fact that they accumulated the virus in their inflorescence tissues to concentrations similar to those observed in the other four NILs that developed severe symptoms (mean scores between 2.4 and 2.8) (Fig. 3). These results confirm the PSI1.2 QTL and demonstrate a major effect of this QTL on the development of symptoms in response to infection by PPV-R. The distinct phenotypes of the NILs LCN1-2.3 and LCN1-2.10 locate PSI1.2 between 0 and $1,471 \mathrm{kbp}$ at the long distal part of chromosome 1 .
PSI1.1 was also confirmed by using a Col $\times$ Ler NIL called SRL1-52-10/1, which contains a Ler introgression between 0 and $24 \mathrm{cM}$ on chromosome 1 in a Col background (Fig. 4). A SYMP score of $2.8 \pm 0.3$ was recorded for this NIL, significantly higher than the symptom score of the Col parent $(1.4 \pm$ $1.1)$, while there was no significant difference in viral accumulation $(42.0 \pm 9 \%$ compared with $52.0 \pm 24 \%)$.

The PSI3 QTL detected in the Col $\times$ Ler population, having a positive additive effect, could be confirmed using the SRL3 NIL, which contains a $10-\mathrm{cM}$ Ler introgression in a Col background (Fig. 4). The PPV-R-infected SRL3 NIL showed a significantly higher SYMP score than that of the Col parent $(3.2 \pm$ 0.4 compared with $1.4 \pm 1.1$ ), despite the fact that virus accumulation, with an ACC score of $45.0 \pm 10 \%$ for SRL3, was not significantly different from that observed in Col $(52.0 \pm 24 \%)$.

To confirm PSI5, the SYMP QTL identified on chromosome 5 with Cvi alleles increasing symptom severity (Table 2), two NILs with a Cvi introgression in a Ler background, LCN5-4 and LCN5-7, were inoculated with PPV-R. LCN5-7, which carries a large Cvi introgression covering the QTL region, displayed extremely severe symptoms (mean SYMP score of 4.0, with 7 of the 11 tested plants ultimately dying from infection, which, in this study, was never observed in the Ler parent). This score is significantly different $(P(f)<0.0001)$ from that for LCN5-4, which carries a smaller, nonoverlapping Cvi

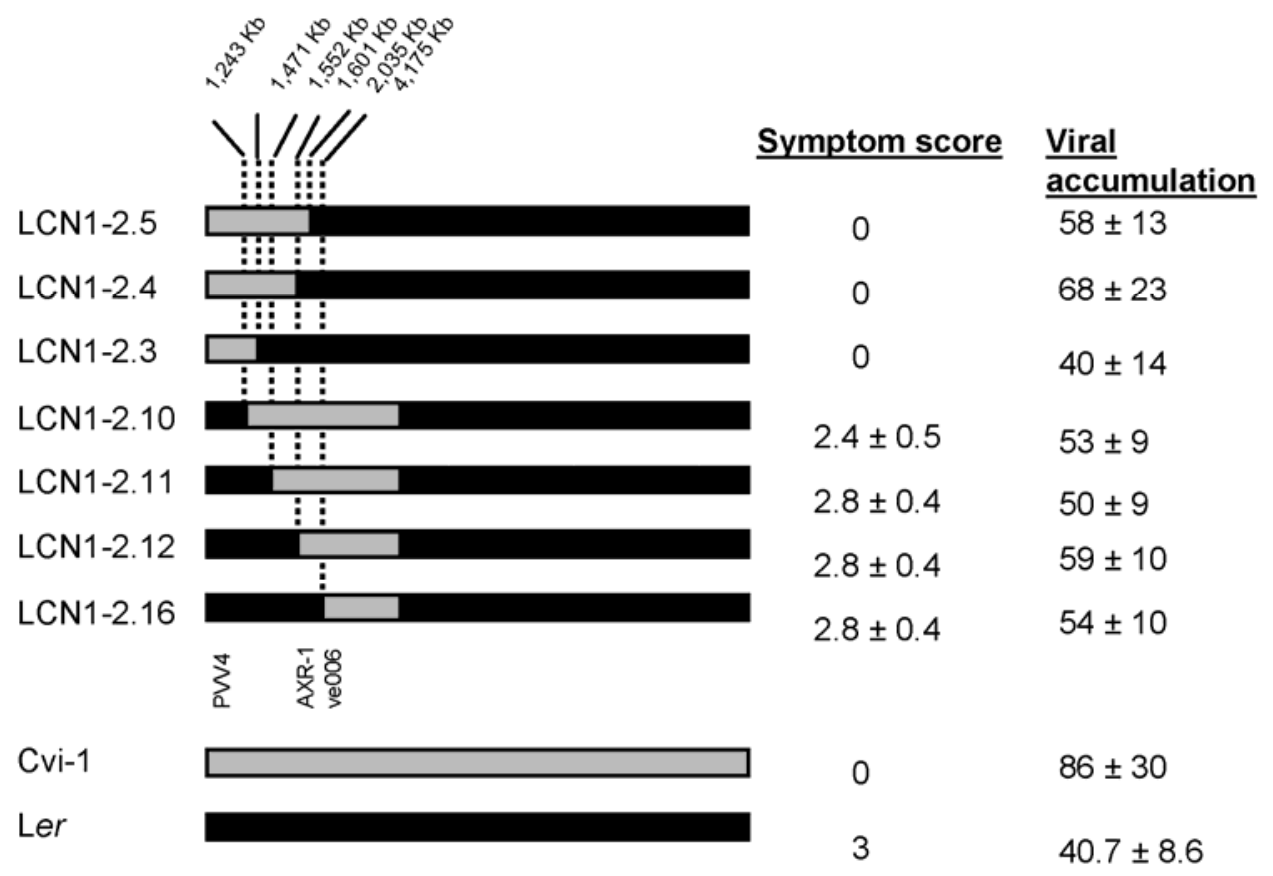

Fig. 3. Schematic representation of near-isogenic lines (NILs) showing chromosome 1 Cape Verde islands (Cvi) introgressions (in gray) in a Landsberg erecta (Ler) background (in black) and their responses to Plum pox virus Rankovic (PPV-R) infection (symptom score and viral accumulation).

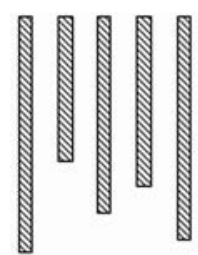

Col-0
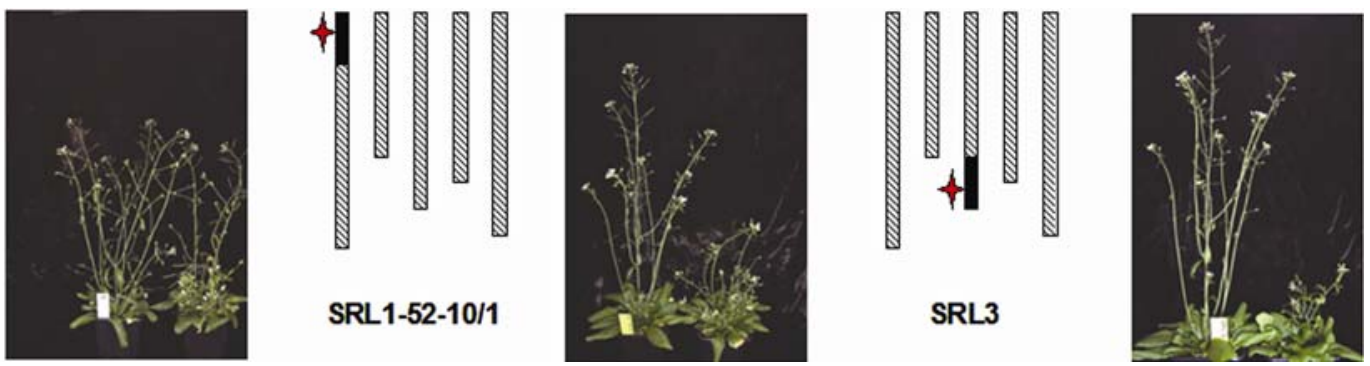

Fig. 4. Representation of two near-isogenic lines (SRL 1-52-10/1 and SRL3) showing chromosome 1 and 3 Landsberg erecta introgressions (in black) in a Col-0 (Columbia) background (in hatched black) and their phenotypic responses to Plum pox virus Rankovic infection, compared with the Col-0 parent. Healthy plants are on the left and infected plants on the right. The red star indicates the approximate quantitative trait loci positions on chromosomes 1 and 3. 
introgression, or for the Ler parent $(3 \pm 0$ and $3 \pm 0.4$, respectively) (Fig. 5). The direct comparison of symptom development in the two NILs thus confirms the PSI5 symptom QTL and demonstrates the additive effect of the Cvi PSI5 allele in a Ler background (Fig. 5), namely a dramatic increase in symptom severity. The opposite effects for the various SYMP QTL explain, at least partly, the transgression observed for the SYMP phenotypic trait values in the Cvi $\times$ Ler F2 and RIL populations.

Since the 2-LOD (logarithm of the odds ratio) ACC1 and $A C C 3$ intervals overlap with the PSII.1 and PSI3 SYMP QTL, the phenotypic analyses of the SRL1-52-10/1 and SRL3 NILs reported above were also used to try to confirm the two $A C C$ QTL. Since, in both cases, viral accumulation in the NILs was not significantly different from that observed in the recurrent Col-0 parent, we were not able to confirm the effect of any of these two QTL. Similarly, the ACC4 QTL was not confirmed in the PPV-R-infected LCN4-3 NIL (Keurentjes et al. 2007).

\section{Resistance determinants in the Cvi $\times$ Ler population.}

In the Cvi $\times$ Ler $\mathrm{F} 2$ population, 12 of the 110 inoculated plants failed to accumulate PPV-R to detectable levels in their inflorescence tissue. In the corresponding RIL population, 24 of the 156 RILs behaved similarly (discussed above), indicating that one or more resistance factors segregate in these populations. A first resistance determinant originating from the Cvi parent was mapped on chromosome 1 between the CH.200C and EC.88C markers, with a LOD score of 4.89 (Fig. 2). Interestingly, it colocalizes with the $r p v 1$ locus, conferring resistance to the PPV-PS isolate, identified by Decroocq and associates (2006) in Cvi. As expected from a recessive resistance model, the Ler (susceptibility) allele at this position is strongly associated with susceptibility (of 88 RILs that have the Ler allele at this position, 85 are susceptible to PPV-R as demonstrated by detectable viral accumulation in the inflorescence tissue). On the other hand, the Cvi allele is not correlated with resistance. Of 46 RILs with the Cvi allele, 25 are susceptible and 21 are resistant, indicating that a second genetic determinant may be needed for resistance. Those data were confirmed by using the frequency of resistant plants in each of the 156 RILs (data not shown). Due to nonnormal distribution of the data, a nonparametric test was performed and showed the closest linkage of $r p v 1$ with the EC.88C marker (significance of $5 \times 10^{-4}$ ).

Using the subpopulation of the 46 RILs carrying the Cvi allele, a second determinant, named $r p v 3$, originating from the Ler parent, was mapped close to the EC-235L genetic marker on chromosome 2, with a LOD score of 5.09. Again, the predictive value was high for the susceptibility allele at this locus (of 56 RILs with the Cvi susceptibility allele, 50 are susceptible) but not for the resistance Ler allele (75 susceptible and 18 resistant). Analysis of the four genotypic classes for the two loci (Supplemental Fig. S3) suggests that the resistance is conferred by the simultaneous action of at least two recessive genes on linkage groups 1 and 2 .

To determine if this resistance is PPV-specific, two resistant and two susceptible RILs were inoculated with two other potyviruses, LMV-AF199 (for which Cvi is resistant and Ler symptomless susceptible) (Revers et al. 2003) and TuMV-UK1 (Cvi and Ler are both susceptible and exhibit symptoms) (Martin Martin et al. 1999). The two resistant RILs carried resistance alleles at both loci controlling PPV resistance but lacked the gene controlling resistance to LMV-AF199 infection described by Revers and associates (2003). The four RILs displayed symptoms upon TuMV-UK1 inoculation, and analysis of viral accumulation indicated that they were symptomless susceptible to LMV-AF199, demonstrating that this recessive, digenic resistance does not confer broad-spectrum protection against potyviruses (data not shown).

\section{DISCUSSION}

In this work, we have analyzed the genetic basis of symptom development during viral infection in A. thaliana. F2 and RIL populations originating from two different crosses were used to evaluate the variability of symptom severity as well as of virus accumulation in Arabidopsis inflorescences infected with the PPV-R isolate. For both traits, the quantitative variations observed were shown to be complex regulated. Quantitative trait analysis allowed the mapping of a number of genetic determinants for both traits in two RIL populations, $\mathrm{Col} \times \mathrm{Ler}$ and Cvi $\times$ Ler.

With respect to symptom severity, two to three QTL were reported, depending on the segregating population, while for virus accumulation, one to three QTL were observed. The effect of each QTL was moderate to medium (explained variance ranging from 5.6 to $17.4 \%$ ), but a high percentage of explained phenotypic variance was observed when considering all the covariants (total explained variance in the $\mathrm{Col} \times$ Ler population is $45 \%$ for SYMP and $38 \%$ for ACC). These relatively low explained-variances values are in agreement with the significant but relatively low heritability values observed for both traits, in particular in the Col $\times$ Ler population. These observations can be explained by several reasons that are not mutually exclusive.

A first one is that the phenotyping strategy used did not allow a sufficiently precise and reproducible evaluation of the quantitative traits under study. In this context, it is noteworthy that the large-scale evaluation of the Col and Cvi parents showed significant plant-to-plant variability, in particular for the ACC trait, as indicated by the large standard deviations obtained. Moreover, from one experiment to another, substantial variations in $\mathrm{ACC}$ values were observed for the parental lines.
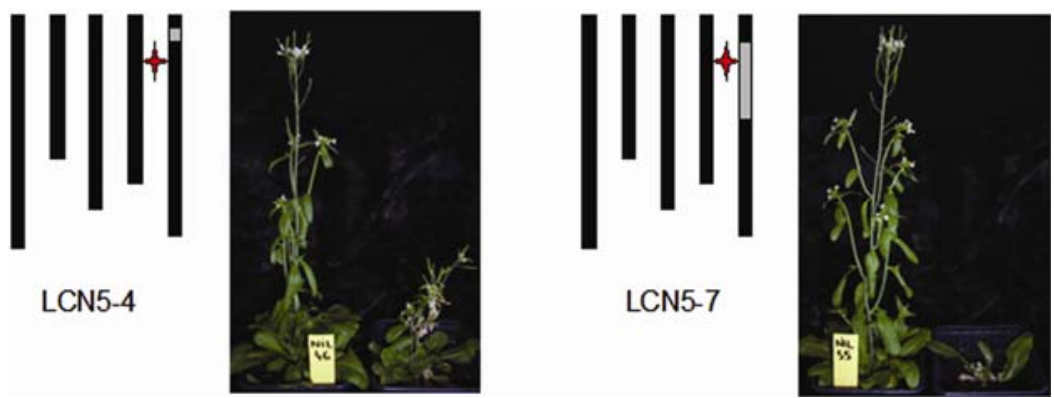

Fig. 5. Representation of two near-isogenic lines (NILs 46 and 55) showing chromosome 5 Cape Verde islands- 1 introgressions (in gray) in a Landsberg erecta background (in black) and their phenotypic responses to Plum pox virus Rankovic infection. For each NIL, healthy plants are on the left and infected plants on the right. The red star indicates the approximate quantitative trait loci position on chromosome 5. 
It is thus conceivable that a phenotyping performed under more stable conditions than those available in the BL3-level containment greenhouse required for PPV manipulation could have allowed a more powerful QTL identification.

A second reason that could account for intermediate level heritability values and low impact of the identified QTL is that these traits are controlled by a larger number of genetic determinants, each contributing only little to the overall trait. The confirmation of some QTL by NILs does not support this hypothesis. However, we cannot rule out the occurrence of many more QTL that we failed to detect because of their small effect.

Whatever the reason or reasons for the moderate to low explained variances of the QTL detected, the involvement of these genetic determinants in the traits studied was confirmed by independent detection in the two different recombinant populations in the case of the PSII locus and the use of NILs for at least two other QTL. Besides PSII, no other QTL were identified in both RIL populations. The simplest explanation is that this could be due to environmental effect, since the populations were tested separately. Three types of QTL-environment interactions have been proposed to explain differential QTL detection while using at least one similar parent ( $\mathrm{Li}$ et al. 2003): i) some QTL are expressed in one environment and not in another, ii) QTL can be expressed more in one condition than in an other, or finally, iii) QTL can have opposite effects in two different environmental conditions. However, another explanation is that different genetic factors are polymorphic in the two crosses or that effects are dependent on genetic background. Similarly, at least two other studies showed contrasting effects of Ler alleles in distinct crosses (El-Lithy et al. 2006; Symonds et al. 2005).

The use of connected crosses has been proposed to increase the genetic variability addressed and to test for epistatic interactions between QTL and the genetic background. Such an approach allows increasing the power of QTL detection, owing to a higher probability that a QTL will be polymorphic in at least one cross. Despite those potentialities, we detected only weak epistatic effect between the above QTL and in one single population, Col $\times$ Ler. Similarly to what was observed in our study, others reported poor QTL congruency when analyzing six connected maize populations (Blanc et al. 2006).

A direct validation of the phenotypic effect of the three symptom QTL, PSII, PSI3, and PSI5 was obtained using NILs.

The location of PSII was narrowed in Cvi-introgression lines to a small segment of approximately $1,500 \mathrm{~kb}$ at the distal part of chromosome 1 . Usually a QTL will account for only a small fraction of the genetic variation as revealed by quantitative analysis in the recombinant populations. This was confirmed in NILs, as shown for PSI3 and PSI5. However, surprisingly, analysis of the introgression lines covering the PSII interval revealed qualitative variation, since PSII alone leads to symptom development upon PPV infection. PSII accounts, therefore, for a major part of the symptom trait and appears to act independently from other loci. However, the low explained variance suggests that symptom development can be diminished by other Cvi or Col loci.

On the contrary, we showed that the presence of the Cvi PSI5 allele in NILs produced more severe symptoms than in the parent Ler. Since Cvi is asymptomatic when infected with PPV-R, we hypothesize that the presence of symptoms requires at least the Ler PSII allele and the Cvi PSI5 allele acts synergistically with $P S I 1$, causing the recombinant lines to exceed the parental phenotypes.

Similarly, the quantitative effect of the PSI3 QTL on symptom severity was demonstrated through the use of lines carrying Ler introgressions in a Col-0 background. Based on the results obtained in both Cvi and Ler introgression lines, we postulate that PSII and the PSI3 or PSI5 are functionally integrated components determining symptom development and severity.

This work also points out another interesting element that concerns the relationship between the two traits studied, symptom development and virus accumulation. It is commonly accepted that a positive correlation exists between fitness and virulence that corresponds to the basic trade-off model (Anderson and May 1982; Ewald 1983; Frank 1992; Nowak and May 1994). In contrast, in our experimental populations, we found little evidence of correlation between virus accumulation and symptom severity, though at least two accumulation QTL, ACC1 and ACC3, colocalize with PSI1 and PSI3, respectively. In the Cvi $\times$ Ler population, no colocalization between SYMP and ACC QTL was detected. Noteworthy, some Cvi $\times$ Ler RILS and NILs consistently presented a high virus titer but no symptoms at all. Three models have been proposed to explain possible association between two traits mapping to the same genetic locus (Lebreton et al. 1995): i) two closely linked QTL control the two traits independently, ii) one single QTL controls both trait, iii) or one QTL controls one trait that is causally associated to the other one. However, we could not rule out one or the other model concerning the QTL controlling symptom severity and virus accumulation on chromosomes 1 and 3 .

Although multiple determinants for symptom development and severity were detected, transgressive segregation resulted in several RILs of the Cvi $\times$ Ler population that were fully resistant upon PPV infection. The data presented here indicate that both parents possess genetic determinants that affect PPV infection. Cvi shows intermediate susceptibility to PPV-R infection, while Ler is fully susceptible. Therefore, the genetic basis for the PPV-R resistance in the Cvi $\times$ Ler RILs is more complex than can be explained by a single-gene model. Our data points out to a recessive resistance conditioned by two unlinked loci, one coming from Cvi and the other from Ler. The nature of this resistance is still unknown. Since initially PPV-R was detected in inoculated Cvi leaves (Decroocq et al. 2006), we hypothesized that this partial resistance was related to an inhibition of PPV long-distance movement. However, in a $r p v 1 / r p v 3$ background, the mechanism leading to the digenic resistance has not yet been described. It should be noted that in two other model plants, tobacco and soybean, a similar digenic, recessive mechanism that restricts virus long-distance movement was described (Goodrick et al. 1991; Schaad and Carrington 1996). For example, Tobacco etch virus is able to establish infection and move from cell to cell in inoculated leaves of tobacco line V20 carrying two unlinked recessive genes, but systemic infection in distal tissues is impaired (Schaad and Carrington 1996). Recently, Ruffel and associates (2006) also identified digenic, recessive determinants controlling the resistance to Pepper veinal mottle virus (PVMV) infection in pepper. But in this case, PVMV is restricted early during infection in the inoculated leaves, and the resistance is linked to the eIF4E and eIFiso4E eukaryotic initiation factors.

Since recessive genes that control these restriction phenotypes are important to dissect the host requirements for virus infection, positional cloning of Arabidopsis genes is currently going on. One of them is mapping in the same region as the previously mapped resistance gene rpvl (Decroocq et al. 2006). $r p v 1$ is controlling the resistance to PPV-PS infection, and we hypothesize that it is also partially controlling infection by PPV-R. The second recessive gene mapped at the bottom of chromosome 2 and is holding from Ler. Neither the rpv1 nor $r p v 3$ locus is colocalizing with the Arabidopsis eIF4E and isoform copies. 
In this study, QTL mapping provided a means of identifying specific host factors associated with symptom development during virus infection. These results will be further coupled with fine-scale mapping, followed by QTL positional cloning. PSIl will be our first candidate. It is also noteworthy that we failed initially to detect PSI1 dominance over other QTL, despite the significant major effect of PSII in NILs. This result illustrates the limit of resolution typical of QTL studies and is even more accurate when detecting loci explaining only a small part of the trait variation.

\section{MATERIALS AND METHODS}

\section{Plant materials.}

F2 and RIL populations derived from the crosses between Col-0 and Ler (Lister and Dean 1993) and between Cvi-1 and Ler (Alonso-Blanco et al. 1998b) were obtained from the Nottingham Arabidopsis Stock Center (NASC) or from the Institute for Agronomy Research (INRA)-Versailles collection. Plants were grown in a BL-3 containment greenhouse under temperature- and humidity-controlled conditions $\left(20^{\circ} \mathrm{C}\right.$ and relative humidity of $60 \%$ ).

A total of 102 F2 individuals of the $\mathrm{Col} \times$ Ler population and six replicates (one mock-inoculated control and five inoculated plants) for each of the 101 RILs derived from this cross were phenotyped following PPV-R inoculation.

Similarly, 110 F2 individuals of the Cvi $\times$ Ler population and five replicates (one mock-inoculated control and four inoculated plants) for each of 156 RILs derived from this cross were also phenotyped consequently to PPV-R inoculation. This experiment was performed using a complete randomblock design.

NILs derived from the Col $\times$ Ler cross and in which Ler genomic regions have been introgressed into a Col-0 background were provided by M. Kearsey (University of Birmingham, U.K.) or were obtained from the NASC (Koumproglou et al. 2002). NILs originating from the Ler $\times$ Cvi cross and in which Cvi-1 genomic regions were introgressed into a Ler background have been developed at the Wageningen Agricultural University (Alonso-Blanco et al. 1998a; Keurentjes et al. 2007). A total of 12 to 24 replicates of each NIL were tested by inoculation with PPV-R.

\section{Virus material.}

The PPV-R isolate is available as an infectious cDNA clone called pICPPV (Saenz et al. 2000). It was initially inoculated by particle-gun bombardment and was then further propagated in $N$. benthamiana. The inoculation of Arabidopsis plants with PPV-R was performed as previously described (Decroocq et al. 2006).

Two other potyviruses were also used: TuMV, available as an infectious clone of the UK1 isolate (Jenner et al. 2000) provided by F. Ponz (Centro de Investigacion y tecnologica del Instituto Nacional de Investigaciones Agraria, Madrid) and propagated on turnip, and LMV-AF199, available in our laboratory (Krause-Sakate et al. 2002) and propagated in lettuce (cv. Trocadéro). Both viruses were inoculated as for PPV.

\section{Phenotyping.}

RIL and F2 plants were monitored for the appearance of symptoms and were scored 15 to $21 \mathrm{dpi}$. PPV symptoms consist of inflorescence distortions with more or less severe stunting. In severe cases, yellowing or browning of the inflorescences is also observed. Scoring of symptom severity was performed using a notation scale ranging from 0 to 4: 0 corresponds to plants in which the virus is present but that show no morphological differences as compared with the healthy plant:
1 to plants with no stunting but showing top incurvation on a single floral hamp, 2 to plants presenting distortions on a majority of hamps but with only a slight reduction of their size, 3 to plants with distortions on all floral hamps and showing a strong growth reduction, and finally, 4 to plants with complete inhibition of floral hamp development and severe stunting of the whole plant (Fig. 1). The scoring of F2 populations and RILs was performed at least twice by two independent evaluators. The final score used is the mean of these independent scorings.

At 21 days after inoculation, virus accumulation in inflorescence tissues was evaluated for each individual plant, using a semiquantitative double antibody sandwich-enzyme-linked immunosorbent assay (ELISA) assay with anti-PPV commercial reagents $(\mathrm{D}+\mathrm{M}$ polyclonal antibody, LCA Laboratory, Blanquefort, France). Optical densities were normalized using a PPV-R infected, $N$. benthamiana positive control deposited on every ELISA plate of an assay. In the case of RILs, the final ACC value is the average of normalized measurements from all PPV-infected replicates of each RIL. In order to obtain more reproducible results and to eliminate potential interference from plant substances, all assays were performed on a 1:100 dilution of crude plant extracts prepared by grinding the plant tissues $(1: 4 \mathrm{wt} / \mathrm{vol})$ in a phosphate buffered saline-Tween polyvinylpyrrolidone (PVP) buffer $(136.9 \mathrm{mM} \mathrm{NaCl}, 1.47 \mathrm{mM}$ $\mathrm{KH}_{2} \mathrm{PO}_{4}, 2.68 \mathrm{mM} \mathrm{KCl}, 8.1 \mathrm{mM}$ de $\mathrm{Na}_{2} \mathrm{HPO}_{4}, 0.05 \%$ [vol/vol] Tween 20, 21\% [wt/vol] PVP 25K). Independently of the evaluation of the viral concentration, this test allowed the confirmation of the presence of the virus in symptomlessly infected plants (0 in SYMP scale), thus excluding potential inoculation escapes.

\section{Statistical analysis.}

Data analysis was performed using the generalized linear model (PROC GLM) of the SAS package (SAS institute, Cary, NC, U.S.A.). ANOVA allowed the determination of the specific effect of 'genotype' and broad-sense heritability, which is the ratio between the genetic variance and the total phenotypic variance and is calculated using the formula:

$h^{2}=\sigma_{g}^{2} /\left[\sigma_{g}^{2}+\left(\sigma_{e}^{2} / n\right)\right]$, where $\sigma_{g}^{2}$ is the genetic variance, $\sigma_{e}^{2}$ is the environmental variance, and $n$ the number of replicates. Frequency distribution and Pearson's correlation coefficients were calculated using the statistical and graphical functions of EXCEL and the statistical software R.

\section{Genetic determinants mapping.}

A set of 91 markers was previously scored in the $\mathrm{Col} \times \mathrm{Ler}$ RIL population as displayed on the NASC website. They were selected following two criteria. i.e., acceptable Chi square and few missing data. For the Cvi $\times$ Ler linkage map, 99 markers were chosen and extracted from the European Natural cooperative database. The RIL-derived genetic maps were constucted using MAPMAKER 3.0 software and the Kosambi map unit function (Lander and Green 1987). MAPMAKER 3.0 was also used to map monogenic determinants in the Cvi $\times$ Ler RIL population.

Both Col $\times$ Ler and Cvi $\times$ Ler linkage maps served as the basis for QTL analysis in QTL Cartographer for MS Windows version 2.5 (Basten et al. 1994; North Carolina QTL Cartographer website). Interval mapping (Lander and Botstein 1989) was first performed to determine putative QTL, and then, composite interval mapping (Zeng 1994) was performed, scanning the genome at 2-cM intervals and using a window size of 10 $\mathrm{cM}$. Five markers were selected as cofactors under the forward regression method. A 1,000-permutations test allowed estimation of the LOD significance threshold for each trait, as described by Churchill and Doerge (1994). A conservative LOD 
score of 2.7 was used as the threshold for detecting QTL significance. The percentage of the phenotypic variation explained by the QTL corresponds to the regression value $R^{2}$ taken at the peak LOD score of the QTL.

In addition, in cases of nonnormal trait distribution, a nonparametric Kruskal-Wallis rank-sum test was performed (Lehmann 1975). An association between trait and molecular markers was considered significant below the $1 \%$ level.

The program PROC GLM was used to perform a two-way interaction ANOVA to detect significant QTL $\times$ QTL epistasis via the corresponding marker $\times$ marker interactions and epistasis.

\section{ACKNOWLEDGMENTS}

Thanks to G. Marandel and S. Decroocq for advice and assistance in QTL mapping, to T. Mauduit for the production and maintenance of plants, to J. M'Baya and S. Robert for their technical assistance during their master training period. O. Sicard is funded by a Ph.D. fellowship from INRA and the Aquitaine regional council. This work was supported by both Plant Health and Plant Genetics divisions of INRA.

\section{LITERATURE CITED}

Alonso-Blanco, C., El-Assal, S. E., Coupland, G., and Koornneef, M. 1998a. Analysis of natural allelic variation at flowering time loci in the Landsberg erecta and Cape Verde Islands ecotypes of Arabidopsis thaliana. Genetics 149:749-764.

Alonso-Blanco, C., Peeters, A. J., Koornneef, M., Lister, C., Dean, C., van den Bosch, N., Pot, J., and Kuiper, M. T. 1998b. Development of an AFLP based linkage map of Ler, $\mathrm{Col}$ and Cvi Arabidopsis thaliana ecotypes and construction of a Ler/Cvi recombinant inbred line population. Plant J. 14:259-271.

Anderson, R. M., and May, R. M. 1982. Coevolution of hosts and parasites. Parasitology 85:411-426.

Basten, C. J., Weir, B. S., and Zeng, Z. B. 1994. Zmap: A QTL cartographer. Pages 65-66 in: Computing Strategies and Software. C. Smith, J. S. Gavora, E. B. Burnside, eds. Fifth World Congress on Genetics Applied to Livestock Production. Guelph, Ontario, Canada.

Ben Chaim, A., Grube, R. C., Lapidot, M., Jahn, M., and Paran, I. 2001. Identification of quantitative trait loci associated with resistance to $\mathrm{Cu}$ cumber mosaic virus in Capsicum annuum. Theor. Appl. Genet. 102:1213-1220.

Blanc, G., Charcosset, A., Mangin, B., Gallais, A., and Moreau, L. 2006. Connected populations for detecting quantitative trait loci and testing for epistasis: An application in maize. Theor. Appl. Genet. 113:206-224.

Callaway, A. S., Huang, Z., and Howell, S. H. 2000. Host suppressors in Arabidopsis thaliana of mutations in the movement protein gene of Cauliflower mosaic virus. Mol. Plant-Microbe Interact. 13:512-519.

Churchill, G. A., and Doerge, R. W. 1994. Empirical threshold values for quantitative trait mapping. Genetics 138:963-971.

Dardick, C. D., Golem, S., and Culver, J. N. 2000. Susceptibility and symptom development in Arabidopsis thaliana to Tobacco mosaic virus is influenced by virus cell-to-cell movement. Mol. Plant-Microbe Interact. 13:1139-1144.

Decroocq, V., Foulongne, M., Lambert, P., Le Gall, O., Mantin, C., Pascal, T., Schurdi-Levraud, V., and Kervella, J. 2005. Analogues of virus resistance genes map to QTL for resistance to sharka disease in Prunus davidiana. Mol. Genet. Genomics 272:680-689.

Decroocq, V., Sicard, O., Alamillo, J. M., Lansac, M., Eyquard, J. P., Garcia, J. A., Candresse, T., Le Gall, O., and Revers, F. 2006. Multiple resistance traits control Plum pox virus infection in Arabidopsis thaliana. Mol. Plant-Microbe Interact. 19:541-549.

Dunoyer, P., and Voinnet, O. 2005. The complex interplay between plant viruses and host RNA-silencing pathways. Curr. Opin. Plant Biol. 8:415-423.

Dunoyer, P., Lecellier, C. H., Parizotto, E. A., Himber, C., and Voinnet, O. 2004. Probing the microRNA and small interfering RNA pathways with virus-encoded suppressors of RNA silencing. Plant Cell 16:1235-1250.

El-Lithy, M. E., Bentsink, L., Hanhart, C. J., Ruys, G. J., Rovito, D., Broekhof, J. L., van der Poel, H. J., van Eijk, M. J., Vreugdenhil, D., and Koornneef, M. 2006. New Arabidopsis recombinant inbred line populations genotyped using SNPWave and their use for mapping flowering-time quantitative trait loci. Genetics 172:1867-1876.

Ewald, P. W. 1983. Host-Parasite relations, vectors, and the evolution of disease severity. Ann. Rev. Ecol. Syst. 14:465-485.
Frank, S. A. 1992. A kin selection model for the evolution of virulence. Proc. R. Soc. Lond. B 250:195-197.

Fujisaki, K., Hagihara, F., Azukawa, Y., Kaido, M., Okuno, T., and Mise K. 2004. Identification and characterization of the SSB1 locus involved in symptom development by Spring beauty latent virus infection in Arabidopsis thaliana. Mol. Plant-Microbe Interact. 17:967-975.

Goodrick, B., Kuhn, C., and Hussey, R. 1991. Restricted systemic movement of cowpea chlorotic mottle virus in soybean with nonnecrotic resistance. Phytopathology 81:1426-1431.

Jenner, C. E., Sanchez, F., Nettleship, S. B., Foster, G. D., Ponz, F., and Walsh, J. A. 2000. The cylindrical inclusion gene of Turnip mosaic virus encodes a pathogenic determinant to the Brassica resistance gene TuRB01. Mol. Plant-Microbe Interact. 13:1102-1108.

Kaneko, Y. H., Inukai, T., Suehiro, N., Natsuaki, T., and Masuta, C. 2004. Fine genetic mapping of the TuNI locus causing systemic veinal necrosis by turnip mosaic virus infection in Arabidopsis thaliana. Theor. Appl. Genet. 110:33-40.

Kasschau, K. D., Xie, Z., Allen, E., Llave, C., Chapman, E. J., Krizan, K. A., and Carrington, J. C. 2003. P1/HC-Pro, a viral suppressor of RNA silencing, interferes with Arabidopsis development and miRNA function. Dev. Cell 4:205-217.

Keurentjes, J. J., Bentsink, L., Alonso-Blanco, C., Hanhart, C. J., Blankestijn-De Vries, H., Effgen, S., Vreugdenhil, D., and Koornneef, M. 2007. Development of a near-isogenic line population of Arabidopsis thaliana and comparison of mapping power with a recombinant inbred line population. Genetics 175:891-905.

Koumproglou, R., Wilkes, T. M., Townson, P., Wang, X. Y., Beynon, J., Pooni, H. S., Newbury, H. J., and Kearsey, M. J. 2002. STAIRS: A new genetic resource for functional genomic studies of Arabidopsis. Plant J. 31:355-364.

Krause-Sakate, R., Le Gall, O., Fakhfakh, H., Peypelut, M., Marrakchi, M., Varveri, C., Pavan, M. A., Souche, S., Lot, H., Zerbini, F. M., and Candresse, T. 2002. Molecular characterization of Lettuce mosaic virus field isolates reveals a distinct and widespread type of resistance-breaking isolate: LMV-Most. Phytopathology 92:563-572.

Lander, E. S., and Green, P. 1987. Construction of multilocus genetic linkage maps in humans. Proc. Natl. Acad. Sci. U.S.A. 84:2363-2367.

Lander, E. S., and Botstein, D. 1989. Mapping Mendelian factors underlying quantitative traits using RFLP linkage maps. Genetics 121:185-199.

Lebreton, C., Lazic-Jancic, V., Steed, A., Pekic, S., and Quarrie, S. A. 1995. Identification of QTL for drought responses in maize and their use in testing causal relationships between traits. J. Exp. Bot. 46:853865.

Lee, J. M., Hartman, G. L., Domier, L. L., and Bent, A. F. 1996. Identification and map location of TTR1, a single locus in Arabidopsis thaliana that confers tolerance to tobacco ringspot nepovirus. Mol. PlantMicrobe Interact. 9:729-735.

Lehmann, E. L. 1975. Nonparametrics. McGraw-Hill, New York.

Li, Z. K., Yu, S. B., Lafitte, H. R., Huang, N., Courtois, B., Hittalmani, S., Vijayakumar, C. H., Liu, G. F., Wang, G. C., Shashidhar, H. E., Zhuang, J. Y., Zheng, K. L., Singh, V. P., Sidhu, J. S., Srivantaneeyakul, S., and Khush, G. S. 2003. QTL x environment interactions in rice. I. heading date and plant height. Theor. Appl. Genet. 108:141-153.

Lister, C., and Dean, C. 1993. Recombinant inbred lines for mapping RFLP and phenotypic markers in Arabidopsis thaliana. Plant J. 4:745750.

Martin Martin, A., Cabrera y Poch, H. L., Martinez Herrera, D., and Ponz F. 1999. Resistances to turnip mosaic potyvirus in Arabidopsis thaliana. Mol. Plant-Microbe Interact. 12:1016-1021.

Nowak, M. A., and May, R. M. 1994. Superinfection and the evolution of parasite virulence. Proc. Biol. Sci. 255:81-89.

Park, S. H., Hur, J., Park, J., Lee, S., Lee, T. K., Chang, M., Davi, K. R., Kim, J., and Lee, S. 2002. Identification of a tolerant locus on Arabidopsis thaliana to hypervirulent beet curly top virus $\mathrm{CFH}$ strain. Mol. Cells 13:252-258.

Revers, F., Guiraud, T., Houvenaghel, M. C., Mauduit, T., Le Gall, O., and Candresse, T. 2003. Multiple resistance phenotypes to Lettuce mosaic virus among Arabidopsis thaliana accessions. Mol. Plant-Microbe Interact. 16:608-616.

Ruffel, S., Gallois, J.-L., Moury, B., Robaglia, C., Palloix, A., and Caranta, C. 2006. Simultaneous mutations in translation initiation factors eIF4E and $\mathrm{eIF}$ (iso)4E are required to prevent veinal mottle virus infection of pepper. J. Gen. Virol. 87:2089-2098.

Saenz, P., Cervera, M. T., Dallot, S., Quist, L., Quist, J. B., Riechmann, J. L., and Garcia, J. A. 2000. Identificatio of a pathogenicity determinant of Plum pox virus in the sequence encoding the C-terminal region of protein P3+6K(1). J. Gen. Virol. 81:557:566.

Schaad, M., and Carrington, J. 1996. Suppression of long-distance movement of Tobacco etch virus in a nonsusceptible host. J. Virol. 70:25562561. 
Symonds, V. V., Godoy, A. V., Alconada, T., Botto, J. F., Juenger, T. E., Casal, J. J., and Lloyd, A. M. 2005. Mapping quantitative trait loci in multiple populations of Arabidopsis thaliana identifies natural allelic variation for trichome density. Genetics 169:1649-1658.

Takahashi, H., and Ehara, Y. 1993. Severe chlorotic spot symptoms in cucumber mosaic virus strain Y-infected tobaccos are induced by a combination of the virus coat protein gene and two host recessive genes. Mol. Plant-Microbe Interact. 6:182-189.

Vance, V., and Vaucheret, H. 2001. RNA silencing in plants-defense and counterdefense. Science 292:2277-2280.

Voinnet, O. 2001. RNA silencing as a plant immune system against viruses. Trends Genet. 17:449-459.

Zamir, D., Ekstein-Michelson, I., Zakay, Y., Navot, N., Zeidan, M., Sarfatti, M., Eshed, Y., Harel, E., Pleban, T., Van-Oss, H., Kedar, N., Rabinowitch,
H. D., and Czosnek, H. 1994. Mapping and introgression of a Tomato yellow leaf curl virus tolerance gene, TY-1. Theor. Appl. Genet. 88:141146.

Zeng, Z. B. 1994. Precision mapping of quantitative trait loci. Genetics 136:1457-1468.

\section{AUTHOR-RECOMMENDED INTERNET RESOURCES}

European Natural database: www.dpw.wau.n1/natural/resources/populations.htm

NASC website: arabidopsis.info/RI_data/full_markers.may2001.xls

INRA Versailles collection: dbsgap.versailles.inra.fr/vnat/

North Carolina State University QTL Cartographer website: statgen.ncsu.edu/qtlcart/WQTLCart.htm 